\title{
The Relationship between Reading Anxiety and Reading Performance: A Comparison Study of Chinese Postgraduates in China and in the UK
}

\author{
Zhiyi Zhu \\ School of Foreign Languages, Guangdong Peizheng College, Guangzhou 510830, Guangdong, China \\ Email: 869578429@qq.com
}

\begin{abstract}
This study investigated three aspects that related to Foreign Language (FL) reading anxiety: the FL reading anxiety levels; the relationship between FL reading anxiety and reading performance; the main sources of FL reading anxiety. With both quantitative and qualitative research method, the total amount of 47 Chinese postgraduates took part in a paper questionnaire including Foreign Language Reading Anxiety Scale (FLRAS) and reading test, Cambridge Advanced English (CAE). According to the FLRAS scores, 12 of them had one-to-one semi-structured interviews. The results shows that most Chinese postgraduates experienced a moderate level of English reading anxiety, and that international students have higher reading anxiety than domestic students. No statistically significant relationship was found between FL reading anxiety and the reading test. After thematic coding analysis, the main sources of English reading anxiety among international students were found to be 'demanding text feature' and 'different class settings in the UK'. For domestic students, limited English exposure was the main source. Recommendations for teachers and for both groups of students are provided, and the limitations of the present study and suggestions for future research are discussed at the end of the paper.
\end{abstract}

Keywords: foreign language reading anxiety, reading performance, Chinese postgraduates

\section{Introduction}

Being the most common Foreign Language/Second Language (FL/SL) learning activity and a highly effective way of improving language command, reading has an important place in classrooms (Nuttall, 2005; Saito, Garza \& Horwitz, 1999). However, teachers and students consider that reading in a FL is a difficult activity because it requires the cooperation between cognition, attention, memory, perception as well as comprehension (Sellers, 2000, p.513).

FL reading can be influenced by many variables. For instance, cognitive variables e.g., reading disabilities, cognitive skills; demographic variables e.g., gender, amount of reading in free time; affective variables e.g., anxiety, attitudes toward the FL (Isler \& Yildirim, 2017). The focus of the current study is FL reading anxiety. As one of the components of affective variables, FL reading anxiety has attracted the attention of many researchers since the end of the 20th century (e.g. Saito et al., 1999; Sellers, 2000; Zin, 2010). FL reading anxiety was proposed as an independent variable related to FL reading (Saito et al., 1999). Researchers, such as Sellers (2000) and Zin (2010) investigated the FL reading anxiety level of various learners. They found that students who had a higher language proficiency tended to have lower anxiety regarding reading. This may indicate that postgraduate students, who normally have a more advanced English level, would probably have lower anxiety than students at an undergraduate level. There are, nevertheless, other factors that could affect reading anxiety. For example, Lien (2016, p.131) argues that FL reading anxiety of Asian advanced English as a Foreign Language (EFL) students were also affected by unique cultural factors and intense academic pressure in university.

EFL is becoming widely used in China and more and more people are learning English in China, which increases the opportunities for students to study aboard. According to the report from the Higher Education Statistics Agency (HESA), over 105,000 Chinese students studied in the UK in 2017-2018. The majority of them, more than 60,000, were postgraduates (2019). Overseas experiences, especially for academic purposes may largely reduce the FL reading anxiety because it provides ample time and opportunities to read in the target language and become more familiar with target language reading activities (Zhao, Guo, \& Dynia, 2013, p.773). For this reason, in general, the FL reading anxiety level of international students is much lower than for those who study at home. However, there is little research into the impact of overseas experiences on FL reading anxiety and its subsequent effect upon comprehension. For this reason, the FL reading anxiety level of both Chinese home postgraduates and overseas postgraduates is worth exploring.

In addition, there has been little discussion during the relationship between FL reading anxiety and FL reading performance among adult language learners. Much of the research into adult learners finds that FL reading anxiety and reading performance are negatively correlated (Saito et al.,1999; Sellers, 2000; Shi \& Liu, 2006; Zin, 2010). This negative 
correlation was found among beginner level US undergraduates (Saito et al., 1999); English speakers of Spanish (Sellers, 2000); non-English major Chinese sophomores (Shi et al., 2006); and Malay low proficiency EFL learners (Zin, 2010). However, other research has revealed contrasting findings (Brantmeier, 2005; Mills, Pajares \& Herron, 2006; Joo \& Damron, 2015; Zhao et al., 2013). For example, a negative correlation was found in any other Chinese level undergraduate students but no correlation was found in Elementary Level II Chinese classes (Zhao et al., 2013), partly supporting the results of Brantmeier (2005) and Mills et al. (2006) that FL reading anxiety does not influence reading performance. While a positive correlation between FL reading anxiety and reading performance was found among second-year Korean FL students, the same study found no correlation among students in other academic years (Joo et al., 2015). To date, it seems that no conclusion regarding the relationship between FL reading anxiety and FL reading performance can be made. Therefore, the current study intends to further research the relationship between FL reading anxiety and FL reading performance.

In order to provide practical suggestions for reducing FL reading anxiety in Chinese advanced EFL learners, anxietyprovoking factors were also explored. Many different sources of FL reading anxiety were proposed in previous research, for instance, unfamiliar scripts, unfamiliar cultures, worry about comprehension (Saito et al.,1999; Zhao et al., 2013).

This study aims to fill the following gaps in our knowledge, (a) the differences in FL reading anxiety between Chinese postgraduates in China and in the UK, (b) the relationship between FL reading anxiety and FL reading performance, and (c) the FL reading anxiety-provoking sources of Chinese students in China and in the UK.

This dissertation is divided into six chapters. The second chapter is the literature review, which reviews previous relevant research findings and considers fruitful areas of research for the present study. The next chapter is methodology, in which the participants, data collection instruments, analysis methods, procedures and ethical considerations are described. The following chapter contains the results, analysis and findings. The fifth chapter is the discussion, where the findings of the current study are compared and contrasted with previous findings, and possible explanations are discussed. The last chapter summarizes the main findings of this study, provides recommendations and describes the limitations of the study.

\section{Literature review}

In this chapter, relevant literature is critically reviewed to provide a comprehensive background for the present study. It includes five parts. The first part includes the definitions of reading in a foreign language and FL reading anxiety, followed by literature about the relationship between FL reading anxiety and reading performance. Research on the level of FL reading anxiety is presented in the third part, while the next part focuses on sources of FL reading anxiety. The final part considers the rationale for the current study against the background of previous research.

\subsection{Reading in a FL and FL reading anxiety}

Reading is usually identified as a silent, invisible, internal process, compared to the other three language skills, listening, writing and speaking (Alderson, Haapakangas, Huhta, Nieminen, \& Ullakonoja, 2014, p.72). Reading consists of two major component processes, lower-level processes and higher-level processes. The former processes contribute to word recognition, syntactic parsing and semantic-proposition encoding. Word recognition, as a fundamental process of reading, is a rapid process which involves recognizing word forms and accessing a mental lexicon, via the dynamic links between graphic and phonological form, semantic and syntactic resources. Syntactic parsing is an integration of meaning information from the analysis of word order, tenses, subordinate construction and modality. Semantic-proposition encoding, also known as 'meaning propositions encoding', is a process of connecting related information through specific units of information (Grabe, 2009). The higher-level processes involve text-model formation, situation-model building, and strategic processing. A text-model formation occurs when readers extract the literal meaning of what the writer intends the readers to understand, a process without 'personal' interpretation. Situational-model building is when the reader's interpretation is based on the circumstances that a text refers to. In this case, the interpretations and understanding of the same text by two readers are likely to be different. Strategic processing represents the awareness of a need to alter processing due to the different goals of reading (Grabe, 2009).

Reading as an interactive activity is not only receiving information from reading materials using the above processes, but also employing prior knowledge, including both existing knowledge and textual information, to predict, hypothesise and confirm the interpretation of the text. It is a two-way process involving an interaction between the bottom-up model and the top-down model. In order for the reading processes to work smoothly, if a deficiency occurs in one model, the other model compensates for the deficiency. For example, the impact caused by unfamiliar new words in the word recognition process may be compensated by a knowledge of the text topic (Samuels \& Kamil, 1998). This interactive model requires both the activation of prior knowledge and rapid and automatic word recognition and other lower-level processes (Eskey, 1998). Therefore, if interactivity between the bottom-up model and the top-down model is inefficient, the text will not be effectively 
understood by the readers.

Due to the inefficient and ineffective understanding of the foreign language reading materials, readers may experience anxiety, depression and pressure when reading in a foreign language. The term used to describe this phenomenon is 'foreign language reading anxiety'. Although there is no fully agreed definition of this term, many researchers, Saito et al. (1999), Zhao et al. (2013), agreed that it is similar to but distinguishable from general Foreign Language Anxiety (FLA). FLA was defined as 'a distinct complex of self-perceptions, beliefs, feelings, and behaviours related to classroom language learning arising from the uniqueness of the language learning process' and this language learning process is usually connected with speaking activity (Horwitz, Horwitz \& Cope, 1986, p.128). However, foreign language reading anxiety as a distinctive anxiety associated with reading activity was first proposed by Saito et al. (1999). A specific scale, Foreign Language Reading Anxiety Scale (FLRAS), was designed for measuring foreign language reading anxiety (Saito et al., 1999).

\subsection{Research on the relationship between FL reading anxiety and reading performance}

Being a complex affective variable influencing reading, FL reading anxiety and its impact has been investigated to determine similarities and differences in instruments, participants and findings in order to identify potential areas of investigation for the current study.

A number of studies have found that there is a negative relationship between FL reading anxiety and FL reading performance (Saito et al., 1999; Sellers, 2000; Shi et al., 2006; Mohammadpur \& Ghafournia, 2015; Zin, 2010).

Based on the Foreign Language Classroom Anxiety Scale (FLCAS), Saito et al. (1999) first designed and used a 20item FLRAS to measure anxiety in FL reading. Using the FLRAS on 383 English undergraduates learning different foreign languages (French, Russian, Japanese), they examined the correlation between reading anxiety and reading performance at a US university. Specifically, they found that if reading in a foreign language was regarded as a difficult activity, readers had a higher probability of experiencing more anxiety in reading, and obtaining lower course grades, and vice versa. They concluded that foreign language reading anxiety and reading performance are negatively correlated (Saito et al., 1999).

Similar results were also obtained by Sellers (2000). A different 21-item anxiety scale, Reading Anxiety Scale (RAS), and a reading comprehension assessment including a recall test and a multiple choice test were used in order to measure reading anxiety level and reading performance. The results from 89 qualified English speaking university students, learning Spanish as a FL indicated that the more anxious the participants, the fewer correct responses they gave on multiple choice and recall, while their less anxious counterparts were more likely to give more correct responses and recall. This demonstrated a detrimental relationship between reading anxiety and foreign language reading performance (Sellers, 2000).

The negative relationship between FL reading anxiety and reading performance was found not only in the English speakers who learn other languages but also in non-English speakers who learn EFL (Mohammadpur et al., 2015; Shi et al., 2006; Zin, 2010). Shi et al. (2006) used Saito et al.'s (1999) version of FLRAS to test the reading anxiety among 211 second year non-English major Chinese undergraduate students, revealing that their anxiety scores were negatively correlated with their reading scores in College English Test Band 4 (CET4) in 2004. In addition, a comparison of results of a 25-item Reading Test Anxiety Scale and a Test of English as a Foreign Language (TOFEL) test revealed a negative relationship in 100 undergraduate students partaking in a general English course in different academic areas at a university in Iran (Mohammadpur et al., 2015). Similarly, 218 first year low proficiency English as a Second Language (ESL) learners of a Malaysian university were invited to respond to the FLRAS, a reading comprehension test and a written recall test. The results of the correlation test indicated that the reading anxiety and reading performance were negatively correlated, which is in line with the findings of previous research (Zin, 2010).

As can be seen from the above research, the negative relationship between FL reading anxiety and reading performance has been established by different researchers using different reading anxiety scales and a variety of participants. However, Zhao et al. (2013) conducted a similar study at a large US public university which found subtle variations. The reading anxiety of 114 Chinese as a FL students from three different course levels, Elementary level I, Elementary level II, and Intermediate level, was measured by the scores of FLRAS (Saito et al., 1999), while their reading performances were the reading scores from three chapter tests and one final exam. The results of the correlation test indicated that reading anxiety was negatively associated with the reading performance among students in other class levels but not among Elementary level II students (Zhao et al., 2013). This study indicated that FL reading anxiety may have a less clear relationship with reading performance, supporting the findings of Brantmeier (2005), Mills et al. (2006) and Joo et al. (2015).

Other rarely used reading anxiety scales, a self-created 10-item anxiety questionnaire and an adapted version of Mathematics Anxiety Scale (MAS) were used by Brantmeier (2005) and Mills et al. (2006), in order to test the reading anxiety scores of English speakers whose foreign language is Spanish and French respectively. No correlation between reading anxiety scores and reading performance was found in either studies, suggesting that reading performance is not 
influenced by reading anxiety (Brantmeier, 2005; Mills et al., 2006).

In addition, Joo et al. (2015) analyzed the relationship between reading tests and the FLRAS scores of 100 non-native speaking Korean learners from first-year to fourth-year Korean classes at a large private university in the US. From these results, a positive correlation was found among the second-year students, while no correlation was found among students in other academic years. The positive correlation is in contrast to the findings of many previous studies.

To summarise, over the past two decades a variety of reading anxiety scales and different reading tests have been used to collect the foreign language reading anxiety scores and reading grades in order to analyse the relationship between these two variables. However, the findings are neither consistent nor conclusive. Therefore, the current study will once again explore the relationship between these two variables: foreign language reading anxiety and reading performance.

\subsection{FL reading anxiety level}

The fact that students learning different foreign languages have different FL reading anxiety levels has been noted. However, most studies found that most students had FL reading anxiety at a medium level (Saito et al., 1999; Sellers, 2000; Zhao et al., 2013; Zin, 2010).

University learners who learnt Japanese, French and Russian as a FL respectively were investigated, and the results revealed that they mostly had a moderate FL reading anxiety level. More specifically, Japanese learners, scoring 56 in FLRAS were most anxious, while the second most anxious learners were French learners, scoring 53.14, and the least anxious learners, scoring 47.65 , were Russian learners, which suggested that foreign language reading anxiety varied relative to the target language (Saito et al., 1999). In addition, the majority (65\%) of university participants who learnt Spanish as a FL were in the mid-anxiety category (Sellers, 2000, p.516). Additionally, US university students who studied Chinese as a FL were identified as having a medium reading anxiety level, with a mean score of 53.56 in FLRAS (Zhao et al., 2013, p.770). In Zhao et al.'s study, students who had studied Chinese in China tended to have the lowest FL reading anxiety, followed by those who had been to China for other non-language learning purposes and those who had not been to China (2013). This suggested that the foreign language reading anxiety might be influenced by previous experience with the target language country. What is more, the majority (74\%) of first-year low proficiency ESL undergraduates in Malay experienced moderate anxiety, scoring between 46.8 and 73.2 on FLRAS (Zin, 2010, p.49).

However, a small number of students were identified as either low FL reading anxiety or high FL reading anxiety in these studies as well. According to Sellers (2000), 17\% of participants who learnt Spanish as a FL were in the low-anxiety group while $18 \%$ of participants were in the high-anxiety group (p.516). In Zin' study, a minority (26\%) of low proficiency ESL freshmen who scored over 73.4 in FLRAS were in the high anxiety group, and it was suggested that this may be due to a lack confidence in their reading ability (Zin, 2010, p.49). What is more, Lu and Liu (2015) investigated 1702 Chinese university participants and found that they experienced low anxiety when reading in English, explaining that no intermediate oral communication with others might be the reason.

In short, the majority of adult participants with different language proficiencies and different first languages experienced FL reading anxiety at a medium level, while fewer language learners experienced low or high reading anxiety for whatever reasons. However, these findings did not confirm the common assumption that higher proficiency students are likely to have lower anxiety in FL reading. In light of this, the current study will examine the foreign language reading level among advanced EFL learners.

\subsection{Sources of reading anxiety}

The following major factors causing students' reading anxiety have been identified. These are unfamiliar scripts and writing systems, unfamiliar cultures, worry about comprehension and experience with the target language country.

Unfamiliar scripts and writing systems interrupt the process of decoding the target language, leading to reading anxiety (Joo et al., 2015; Saito et al., 1999; Zhao et al., 2013). In other words, readers may experience higher reading anxiety if the written language they decode belongs to different writing systems, while they may have lower anxiety if the language belongs to the same writing systems. For example, English speakers were less anxious when decoding an alphabet system language such as French, as opposed to Japanese (Saito et al., 1999, p.212). This could be explained by the different activation of the reading processes in relation to the different writing systems. Chinese and English are two typical languages that belong to two different writing systems, the logographic writing system and the alphabetic writing system. In terms of the word recognition, the phonological process is activated after the recognition of orthographic characters when reading Chinese, while the phonological process is directly activated when reading English (Perfetti \& Harris, 2013; Sasaki, 2005). In this way, for Chinese EFL learners, the different word recognition process may be activated in the limited working memory when reading English, which may induce anxiety. 
Unfamiliar cultural concepts mean that a reader has an incomplete knowledge of the target language meanings. This is caused not only by a lack of related topical knowledge but also a lack of familiarity with connections between current and historical knowledge and meanings (Saito et al., 1999; Zhao et al., 2013). Even when readers understand the meaning of every single words, they may experience suspended anxiety as a result of misunderstanding the text, because they cannot understand the deeper meaning of the text (Al-Shboul, Ahmad, Nordin \& Rahman, 2013; Saito et al., 1999; Zhao et al., 2013).

Worry about comprehension is worry which is usually aroused in a test situation. This may occur because of the time limitations and working memory shortages. Readers may be afraid that their understanding of the reading text may be different from their teacher's understanding and other reader's understanding (Zhao et al., 2013).

Readers with target language country experience tended to have less anxiety than those without this experience because they had more opportunities and time to be exposed to the target language (Aida, 1994; Zhao et al., 2013). What is more, with overseas experience, readers for academic purposes were more likely to experience lower anxiety than those reading for non-academic purposes, because academic reading required them to be very familiar with reading activity (Zhao et al., 2013). Thus, readers may have higher reading anxiety if they did not have target language country experience or were not familiar with the reading activity.

\subsection{Research questions}

In light of the inconclusive conclusions regarding the relationship between reading anxiety and reading performance, the inconclusive evidence regarding the foreign language reading level, and a lack of research comparing the foreign language reading anxiety of international students and domestic students, the present study explores the relationship between foreign language reading anxiety and foreign language reading performance among Chinese postgraduates in China and Chinese postgraduates in the UK, and the foreign language reading anxiety levels of the target students. In addition, the sources of foreign language reading anxiety among the target students are also investigated. The three research questions are:

(1) Is there any difference in FL reading anxiety between international students and home students?

(2) Is there a significant relationship between FL reading anxiety and Cambridge English: Advanced (CAE) reading of the international and home students?

(3) What are the possible causes of reading anxiety?

\section{Research methods}

\subsection{Participants}

There were 47 Chinese postgraduate students attended in total. Participants were divided into two groups according to the location of their universities. 23 postgraduates who studied in the UK were labelled Group 1 while 24 postgraduates who studied in China were Group 2.

In order to reduce irrelevant influence caused by the course settings, this study used a convenience sampling method (Cohen, Manison and Morrison, 2011, p.156) to recruit students enrolled in a Teaching English to Speakers of Other Languages (TESOL) course at a UK university and students enrolled in English Education course at a Chinese university.

\subsection{Instruments}

A mixed data collection method was employed in order to collect more comprehensive data, increasing the reliability and validity of the research findings (Biesta, 2017; Robson and McCartan, 2011).

\subsubsection{Background information questionnaire and FLRAS}

The first part of the questionnaire is a background questionnaire. Closed questions were designed to collect participant's nominal information, including gender; age; age of starting learning English; current major; current academic year; previous travel purpose to English-speaking countries, and the length of the trip.

The second part of the questionnaire is a version of Saito et al.'s (1999) Foreign Language Reading Anxiety Scale (FLRAS). It is a Likert scale designed to test a specific anxiety-foreign language reading anxiety. Various aspects of reading anxiety were detected by this 20-item scale, including a) self-reports of various aspects of reading anxiety; b) perceptions of reading difficulties in the target language; c) perceptions of reading skill compared to other foreign language skills. The score of this scale was from 20 to 100 . The higher the scores, the more serious the anxiety.

The words, French, Japanese and Russian in the original version were replaced by the word, English, because the scale tests students' English reading anxiety in this study. Both background information questionnaire and FLRAS are in English but some Chinese translations were also used in response to feedback from the pilot study. 


\subsubsection{CAE reading test}

The CAE reading test used in the current study was copied from the Cambridge University official academic examination papers in 2014 because it had a specific part on reading (Paper 1) with a precise time instruction ( 75 minutes), which was a better vehicle for testing reading performance. In the reading paper, a reading passage has 550-580 words and approximately 3000 words in total. It contains 34 questions and four parts. Part 1 (multiple choice, six questions), Part 2 (gapped text, six questions), Part 3 (multiple choice, seven questions) receive two marks per correct answer, while Part 4 (multiple matching, 15 questions) receive one mark per correct answer (Coward, 2007, p.15). In total, there are 53 marks before weighting.

\subsubsection{Interviews}

The interview was designed to explore the possible factors causing foreign language reading anxiety. Interviewees would review anxiety-inducing factors with the guidance from the interviewer. The interview schedule consisted of three parts and each part progressed from the previous step (Breakwell, Smith and Wright, 2011, p.374). Each interview commenced with the interviewee reviewing the reading difficulty of the given reading test and their feelings towards it, allowing an investigation of affective factors. Based on their responses, the interview moved towards reading anxiety and its level, narrowing the focus of the interview and making it more specific. The causes of reading anxiety were then explored which is the real purpose of the interview. For Group 1 interviewees who is experiencing overseas learning, an extra question about their attitudes towards overseas learning experience was included. In addition to these scheduled questions, questions helping clarification and explanation were sometimes asked in order to help the interviewer and the interviewee to understand each other accurately.

Interviewees included three low-anxiety participants and three high-anxiety participants in each group, according to their FLRAS scores. Participants whose FLRAS scores was less than 46.7 were labelled as low anxiety participants, while those whose FLRAS was higher than 73.4 were labelled as high anxiety (Zin, 2010, p.49). Due to the limited number of participants in the current study, participants whose FLRAS scores were close to these cut-off points were invited to be interviewees, in order to provide a relatively comprehensive sketch of reading anxiety factors. Interviewees were given pseudonyms.

To allow a more flexible approach for interviewees, interviews were conducted via video call and were audio recorded for further analysis. Both the interviewer and interviewees used their first language-Chinese, in conducting the interviews. Although they were advanced English learners, given the short length of the interview, it was more convenient and efficient for interviewees to express their real and authentic opinions in their native language. Using Chinese in the interview avoided many misunderstandings due to participants' limited English vocabulary, thus improving the accuracy and reliability of participants' responses. Additionally, speaking in Chinese encouraged interviewees to go into more detail which allowed for greater depth. However, it was suspected that interviewees might say what they thought the interviewer wanted to hear instead of their real thoughts. In order to reduce this effect, the interviewer did not judge any responses and stayed neutral during the interviews (Cohen et al., 2011, p.425).

\subsection{Procedures}

\subsubsection{Pilot study}

In the pilot study, one participant in each group underwent the same procedure that other participants would be required to do in the actual data collection procedures. It was reported by the pilot participants that in the FLRAS, the meaning of the word 'intimidated' was unknown. Therefore, the Chinese translation, 恐吓, 惊慌, was added in order to prevent misunderstanding of this word, which improved the clarity of this item in the questionnaire.

As for the reading test, it was reported that the difficulty level might be higher than tests taken previously. However, there were three reasons for sticking with this test. Firstly, the English grades in this CAE test map on to the B2 to C2 level of CEFR (Cambridge University Press, 2012), which means that readers should understand the main idea of the long text and its implicit meanings. As the target participants have all been learning English for many years and they were doing a masters programme, it is reasonable to deduce that they have an advanced or near-advanced reading ability, which matches the ability required by the CAE test. In other words, the test was regarded as appropriate and suitable for the reading performance of participants. In addition, the CAE test is not as popular as other language tests such as IELTS and TOEFL for Chinese EFL learners, therefore, it was less probable that the test had been done by the participants, which helped to guarantee the fairness of this test and persuasiveness and credibility of the reading grades. Thirdly, this test avoids marker subjectivity as every question has a standard answer in the reading test. It goes a long way to ensuring fairness and impartiality without marker's subjective emotions. For the above reasons, the CAE reading test was used to test participants' reading performance in the current study.

As for the interview, it was suggested that asking simple and direct questions might reduce the possibility of confusing 
the interviewees. The scheduled questions then be revised before the data collection procedure.

\subsubsection{Data collection procedure}

Following the revision of the research instruments as a result of feedback from the pilot study, data collection proceeded. Firstly, enough participants for the study were recruited. Home students studying in English Education in a Chinese university were recruited by a postgraduate studying on the same course. International students studying in TESOL from a university in the UK, were recruited by the researcher directly via e-mail. 24 students in China and 23 students in the UK consented to take part in this research.

After recruitment, the questionnaire and the reading test were administered in the following manner. Participants in China were managed by the English Education postgraduate while participants in the UK were directly managed by the researcher. Before the official data collection process, participants were informed about their right to withdraw, and signed the consent form. They were then required to fill out a background questionnaire and complete FLRAS independently.

The CAE reading test was distributed to participants until all questionnaire papers had been reclaimed. Participants were asked to finish the reading test in no more than 75 minutes. Answers written after the time limit were not recorded.

After completing these two sections, the scores of FLRAS and the CAE test were calculated by the researcher. Based on the scores of FLRAS and the anxiety classification of Zin (2010), participants who were of low anxiety or high anxiety were invited to a one-to-one semi-structured interview. Before the interviews, interviewees were reminded that the whole interview process would be audio recorded but they could withdraw at any time during the interview. They were also told that they had the possibility to review the written transcripts of their interviews.

\subsection{Data analysis}

All quantitative data was analysed using Statistical Program for Social Science (SPSS), version 25.0.

As for the qualitative data from 12 interviews, the audio records were first transcribed in Chinese and a thematic coding method was used to code the transcriptions, in order to discover the sources of FL reading anxiety among the participants. Quotes from the transcription are translated into English when used in this paper.

\section{Results and analysis}

\subsection{The differences of FL reading anxiety between international students and home students}

\subsubsection{The analysis of students' FL reading anxiety level in each group}

Based on the FLRAS scores, participants who scored below 46.7 were placed in the low anxiety category, while those who scored above 73.4 were in the high anxiety category. The remaining participants were placed in the moderate anxiety category (Zin, 2010, p.49). The distribution of participant' FL reading anxiety, according to anxiety category, is presented in Table 1 below.

Table 1. Proportion of FL reading anxiety level of two groups

\begin{tabular}{ccccc}
\hline \multirow{2}{*}{ Anxiety level } & \multicolumn{2}{c}{ G1 } & \multicolumn{2}{c}{ G2 } \\
\cline { 2 - 5 } & $\mathrm{N}$ & Percentage & $\mathrm{N}$ & Percentage \\
\hline Low & 0 & 0 & 2 & 8.3 \\
Medium & 21 & 91.3 & 21 & 87.5 \\
High & 2 & 8.7 & 1 & 4.2 \\
Total & 23 & 100 & 24 & 100 \\
\hline
\end{tabular}

Note: $\mathrm{G} 1=$ Chinese students in the UK; G2= Chinese students in China.

Table 1 shows that differences in the distribution of reading anxiety levels between the two groups were small. Most participants in the UK (91.3\%) and in China (87.5\%) were in the medium anxiety category, which was far higher than those in other anxiety categories. The majority of Chinese postgraduates experienced moderate FL reading anxiety. Very few participants $(\mathrm{N}=5)$ were placed in the high anxiety category or low anxiety category.

\subsubsection{The difference and similarity of students' FL reading anxiety between international and home students}

In order to explore if the reading anxiety differed between the two groups, the independent samples t-test was applied. It compared the means of the two groups of participants because the FLRAS scores were normally distributed. The independent variable is the major variable, while the dependent variable is the FLRAS scores. Table 2 presents the results of the independent samples t-test. 
Table 2. Independent samples t-test of students' FLRAS scores

\begin{tabular}{ccccccc}
\hline Group & $\mathrm{N}$ & Mean & SD & $\mathrm{t}$ & $\mathrm{p}$ & $\mathrm{df}$ \\
\hline G1 & 23 & 59.57 & 7.71 & 1.20 & .24 & 45 \\
G2 & 24 & 56.83 & 7.94 & & & \\
\hline
\end{tabular}

Note: $\mathrm{G} 1=$ Chinese students in the UK; G2= Chinese students in China.

As Table 2 shows, Group 1 students $(\mathrm{M}=59.57, \mathrm{SD}=7.71)$ had slightly higher FLRAS scores than Group 2 student $(\mathrm{M}=56.83, \mathrm{SD}=7.94)$, indicating that, in general, students in the UK tended to experience higher FL reading anxiety than those who studied at home. However, participants who scored between 46.7 and 73.4 on FLRAS were identified as medium anxiety readers (Zin, 2010, p.49). Therefore, in general, both groups of students experienced medium anxiety when reading English (Table 2). What is more, the mean score for the international students on the variable ' the scores of FLRAS' $(\mathrm{M}=59.57, \mathrm{SD}=7.71)$ did not differ statistically significantly $(\mathrm{t}=1.20, \mathrm{df}=45$, two-tailed $\mathrm{p}=.24)$ from that of the domestic students $(\mathrm{M}=56.83, \mathrm{SD}=7.94)$, indicating that the two groups of students experienced similar foreign language reading anxiety.

Although there was no difference between the reading anxiety level of the two groups in general, in order to conduct an in-depth exploration of participant's perceptions and attitudes on reading anxiety, the Mann-Whitney U test was conducted because the data was nonparametric. Four items with significant differences and similarities are extracted and presented in Table 3.

Table 3. Proportion of agreement on each item of each group

\begin{tabular}{|c|c|c|c|c|c|c|c|}
\hline Items & & $\mathrm{SA} / \mathrm{A}$ & $\mathrm{N}$ & $\mathrm{SD} / \mathrm{D}$ & M & $\mathrm{SD}$ & $\mathrm{p}$ \\
\hline \multirow{2}{*}{ 12. I enjoy reading English. } & G1 & 26.1 & 34.8 & 39.1 & 3.13 & 0.82 & $.00^{*}$ \\
\hline & $\mathrm{G} 2$ & 75.0 & 20.8 & 4.2 & 2.00 & 0.83 & \\
\hline \multirow{2}{*}{ 13. I feel confident when I am reading in English. } & G1 & 34.8 & 20.4 & 34.8 & 3.00 & 0.85 & $.05^{*}$ \\
\hline & $\mathrm{G} 2$ & 54.2 & 37.5 & 8.3 & 2.50 & 0.72 & \\
\hline \multirow{2}{*}{$\begin{array}{l}\text { 20. You have to know so much about English and culture in order to } \\
\text { read English. }\end{array}$} & G1 & 39.1 & 17.4 & 43.5 & 3.04 & 1.07 & $.01 *$ \\
\hline & $\mathrm{G} 2$ & 62.5 & 29.2 & 8.3 & 3.83 & 0.96 & \\
\hline \multirow{2}{*}{ 14. Once you get used to it, reading English is not so difficult } & G1 & 82.6 & 13.0 & 4.3 & 2.09 & 0.67 & .21 \\
\hline & $\mathrm{G} 2$ & 87.5 & 12.5 & & 1.83 & 0.64 & \\
\hline
\end{tabular}

Note: ${ }^{*} \mathrm{p}<.05 ; \mathrm{SA} / \mathrm{A}=$ strongly agree and agree; $\mathrm{N}=$ neither agree nor disagree; $\mathrm{SD} / \mathrm{D}=$ strongly disagree and disagree; $\mathrm{G} 1=\mathrm{Chinese}$ students in the UK; G2= Chinese students in China.

The Mann-Whitney U test was used to determine if there was any statistically significant difference of each item in FLRAS between the two groups. A statistically significant difference was found on items $12(p=.00), 13(p=.05)$ and 20 $(\mathrm{p}=.01)$ (Table 3$)$.

According to Table 3, items 12 and 13 describe feelings towards English reading. As for item 12 'I enjoy reading English', two groups of participants held completely different opinions $(\mathrm{p}=.00)$. Three quarters of domestic students believed that reading English is a pleasant and enjoyable activity, while only one quarter of international students agreed with this statement. Meanwhile, nearly two fifths of students (39.1\%) in the UK had the opposite opinions that he or she 'did not enjoy reading English', but only one student in China (4.2\%) held this opinion.

Apart from different attitudes regarding the pleasure that reading brings, statistically significantly different attitudes regarding the confidence they experienced during English reading $(\mathrm{p}=.05)$ were found. Nearly one thirds of international students (34.8\%) while over half of the domestic students (54.2\%) were confident in English reading. On the contrary, 34.8\% of international students were not confident when they read English, while only $8.3 \%$ of students in China lacked confidence in English reading. With regards to items 12 and 13, students in China tended to have a more positive and optimistic attitude towards English reading than their counterparts in the UK, which may be the reason why domestic students had lower reading anxiety scores.

What is more, a statistically significant difference was found on item 20, the importance that English history and culture played in English reading ( $\mathrm{p}=.01) .62 .5 \%$ of domestic participants agreed that English history and culture were beneficial to English reading, far exceeding their international counterparts $(39.1 \%)$. In contrast, nearly half of the participants in the UK (43.5\%) reckoned that English history and culture did not play an important role in English reading, compared to $8.3 \%$ of participants in China. Therefore, this shows that the knowledge of English history and culture was regarded as important 
for students in China.

On the other hand, both groups had similar attitudes towards item 14 . No fewer than $80 \%$ of students from both groups agreed with the statement that reading English is much easier once it becomes a habit, which suggests that most participants believed that anxiety could be reduced if reading in English became a regular and routine activity.

In terms of the first research questions, international students had slightly higher FLRAS scores than domestic students, probably because they were not enjoying as much and not as confident as domestic students during English reading. However, the independent samples t-test shows that two groups of students experienced similar moderate foreign language reading anxiety in general (Table 2).

\subsection{The relationship between reading anxiety and CAE reading of international and home students}

In order to explore the correlation between two variables in each group, the correlations of each group are presented in different tables.

Table 4 and Table 5 illustrate the results of the correlation between reading anxiety scores and the reading scores separately.

Table 4. Correlation between FLRAS scores and CAE reading grades of Group 1

\begin{tabular}{cccc}
\hline Measure & & FLRAS scores & CAE reading grades \\
\hline FLRAS scores & Correlation coefficient & - & -.29 \\
& Sig (2-tailed) & & .18 \\
CAE reading grades & Correlation coefficient & -.29 & - \\
& Sig (2-tailed) & .18 & \\
\hline
\end{tabular}

Using Spearman's correlation, no statistically significant correlation was found between reading anxiety and reading grades among students studying in the UK $(r=-.29, \mathrm{p}=.18)$. In other words, international students' reading anxiety did not influence their reading performance (Table 4).

Table 5. Correlation between FLRAS scores and CAE reading grades of Group 2

\begin{tabular}{cccc}
\hline Measure & FLRAS grades & CAE reading grades \\
\hline FLRAS scores & Pearson correlation & - & -.26 \\
& Sig (2-tailed) & & .22 \\
CAE reading grades & Pearson correlation & -.26 & - \\
& Sig (2-tailed) & .22 & \\
\hline
\end{tabular}

Pearson Product Moment correlation found that there was no statistically significant correlation found between FLRAS scores and CAE reading grades $(r=-.26, p=.22)$, which indicated that the reading performance of home students may not have been influenced by reading anxiety (Table 5 ).

To summarize, there was no statistically significant correlation between FLRAS and CAE grades either in international students or home students, which suggested that foreign language reading anxiety may have had no effect on reading performance (Table 4; Table 5).

\subsection{The possible sources of reading anxiety}

Thematic coding was used to analyse the interviews. The results show that 'fear of negative evaluation' and 'unknown test grades' were two common sources causing reading anxiety. Two other reasons 'demanding text feature' and 'different class settings' caused foreign language reading anxiety among international students, while 'limited English exposure’ was the main source of anxiety for many students in China.

\subsubsection{Students in the UK \\ 4.3.1.1 Demanding text features}

Compared with students in China, it is assumed that international students in the UK may have lower reading anxiety because they are living in an English speaking country and have more opportunities and places to get in touch with the English language, such as menus and references (Zhao et al., 2013). Various types of reading activity may encourage the feeling that reading English is a part of their daily life, thus lowering anxiety. However, they disagreed with this assumption and reported that these reading opportunities required higher English reading ability, creating more pressure and anxiety. These opportunities involve the use of vocabulary and the complex sentences. Firstly, vocabulary may be formal, abstract 
or jargon. Therefore, when they encounter new words frequently, they will experience anxiety in reading. As Louis said,

For me, the language environment forces me to learn, to acquire, and to use English in the UK, but English in reading differs from English in speaking. Compared to my previous university life, I have done much more required reading since the pre-sessional course. It is difficult due to the frequency of new terminology. At that time, I cannot understand the overall meaning of a sentence, even if I can understand the meaning of every single words. In terms of the meaning of words, I have to use the e-dictionary on my phone when I read a menu because there are too many uncommon words about food for me. (Louis)

Similarly, complicated and elaborative structures used in written materials to improve the logic and sophistication of sentences, is distinct to those active and short, or incomplete sentence structures commonly used in communication because delivering simple messages may be the main purpose of speaking. This distinction might increase the more time they spend reading and understanding the texts, which might aggravate reader's anxiety. As Lucy remarked,

The worst thing about reading is encountering long and complicated sentences. You have to sort out the sentence components, remember word order and figure out the referent of the pronouns, which is time consuming. It is even worse with some unknown vocabulary because I know neither the meaning of the vocabulary nor the structure of the sentence. I may forget what I read after sorting out the latter part of the sentence, so I have to read the whole sentence again, which is annoying. This difficulty may be because I do not have enough reading experience in this genre. On the other hand, it is easier to communicate orally because difficult words are seldom used in conversation. However, some expressions are common for natives, such as 'let me know' instead of 'please tell me', but I still prefer to say the latter one. I guess it is what I am used to. (Lucy)

In this way, complicated vocabulary and interlaced sentences causing demanding text feature could provoke feelings of anxiety among international students.

\subsubsection{Different class settings}

Over half the international interviewees stated that the length of the academic year, the learning styles and the teaching approaches in China and in the UK are different. The academic year of most postgraduate programmes in UK universities is one year, while it is two or three years in China depending on the types of the programme. The length of the programme may influence the curriculum settings and designs, and students' learning tasks. As Linda mentioned, intensive learning task in a year raised her anxiety when reading.

One possible reason is that the course in the UK is relatively intensive, as it lasts one year only. In China, lessons are quite relaxed during term time. It is a big challenge for me to finish so many things in this year. My daily routine is doing the required reading before a lecture, and doing required reading after this lecture...(Linda)

What is more, students in the UK are required to do self-study stage before classes. Understanding and consolidation of related knowledge, as well as sorting out questions is furthered via discussions in classes. Therefore, reading ability plays a very important role in the English learning style. However, the previous learning culture of these students was very different. The importance of reading was low or even non-existent. Thus, the change of the role that reading played in learning might have provoked pressure and anxiety. In addition, the teaching approach in the UK is student-centred, which requires not only reading ability, but also the abilities of critical thinking, interpreting, proposing questions, sharing opinions and solving problems. Chinese students might lack these abilities because they stayed in a teacher-centred class environment and highly depended on teachers for a long period. Thus, the change from a teacher-centred teaching approach in China to a studentcentred teaching approach might cause anxiety when reading. As Lydia said,

To be honest, I felt very stressed when I had classes, especially in term 1. I had to do the pre-reading before every lesson. If I did not finish the pre-reading, I felt anxious during the class because I was not sure whether I could follow the lesson. I think UK teachers hope students will show evidence of critical thinking during the discussion and share it with classmates. It seems to be a way to measure my work on reading and I am not very confident about it. In China, students listen and follow teachers'instructions in the classroom, and are seldom called on to answer questions. (Lydia) 
Hence, adapting to a change in the class settings in a short period of time might be one of the factors causing reading anxiety for international students.

\subsubsection{Students in China}

Limited English exposure: Almost all Chinese EFL learners lack an immersive English language environment, as an important cause of anxiety suggested by Group 2 students. Limited English exposure includes two aspects, a limited amount of reading input and low requirements of output. Firstly, for students in China, their English reading input is mainly in class and this is followed by a post-reading activity. English reading is unlikely to be considerable or effective due to limited class time, and the ineffectiveness and limited assessment of post-reading. Thus, effective English reading input for domestic students is inadequate and incomplete. In addition, as the interviewees mentioned, they do not have the opportunities to use oral English because the use of English in a Chinese person's daily life is limited. The essay is probably the only way that reading is assessed. That is why postgraduates in China have lower English reading requirements than students in the UK. Insufficient reading input and lower assessment requirements may be an important cause of reading anxiety among domestic students. Three Group 2 interviewees stated,

As for the input of English, I would say it comes from the classrooms mostly, such as Linguistics, and Lesson Planning and Implementation. However, not all lessons were taught in English. We were required to write our assignments in English but most of the articles I read were in Chinese because of my slow reading speed. Therefore, I felt frustrated when I took a long time to read a 20-page article. However, I like watching English videos from Bilibili (a Chinese video sharing application), but English is not useful in daily conversation because of its unpopularity. I watch American series as well, but I just watch it. No one talks to you in English here. (Anita)

\section{Discussion}

\subsection{A comparison of FL reading anxiety in international students and home students}

According to the analysis and comparison of the FLRAS scores, the following conclusion can be drawn: the two groups of students had a similar level of reading anxiety, a medium level. This result is in line with the findings of Saito et al. (1999), Sellers (2000), Zhao et al. (2013) and Zin (2010). Their studies show that the majority of foreign language learners experienced a moderate level of reading anxiety.

These results without the statistically significant difference in reading anxiety can be explained by the long-term similar educational backgrounds of both groups of participants and their postgraduate programmes. Chinese citizens are obliged to attend primary schools when they are 7. English has been taught as a compulsory course since 2001 (Ministry of Education of The People's Republic of China, 2001). Nearly all of the participants are at a similar age and started learning English at primary school level or even younger, which means that they had been learning English for more than 15 years for both mandatory education and further education. For example, all of them were required to learn English in preparation for the College Entrance Examination that includes an English test for undergraduates. All undergraduates were required to have English classes in order to pass the CET4 as a basic requirement for their graduation. Thus, all Chinese undergraduates are required to have a basic English level due to these mandatory requirements. While for the postgraduate programme, students in Group 1 had to achieve an entry score in IELTS to prove their English proficiency level, while participants in Group 2 had to pass the National Examination Test for MA/MS Candidates (NETM) on a related subject, which includes an English test and also requires a higher English level. In this way, participants in both groups had to achieve a relatively advanced English level because of the requirements for both mandatory education and further education. What is more, the postgraduate courses of participants in both groups are similar which suggests that they have a substantially similar postgraduate curriculum. Thus, it is reasonable to assume that the prolonged similar English learning background and postgraduate curriculum of these two groups led to an equivalence in foreign language reading anxiety.

Although the two groups in the current study experienced similar anxiety levels, international students had higher FLRAS scores, indicating higher foreign language reading anxiety. This is inconsistent with the findings of most previous research demonstrating that students with overseas experience, especially for academic purpose, should have lower reading anxiety because they have more time and opportunities to become familiar with the target language and its cultures (Aida, 1994; Joo et al., 2015; Zhao et al., 2013; Zhou, 2017). EFL learners feeling confident and satisfied regarding English reading probably experience less English reading anxiety (Lien, 2016, p.132). However, according to the results of the MannWhitney U test, international students did not enjoy as much and were not as confident as their domestic counterparts. Based on the interviews, two possible explanations for their low enjoyment and low confidence in reading suggest themselves. One explanation is the change of reading purpose and the high frequency of demanding English input. Interviewees generally 
believed that the reading purpose in classes in China was scanning information, while in UK classes, it was believed to be thinking, interpreting and internalizing knowledge. Therefore, reading becomes a more demanding activity for international students because being an audience is no longer their main role in the class in the UK. Apart from the change of reading roles in class, the frequency of English input was higher. Having more opportunities to read English both in and out of classes, international students should become more familiar with English reading activities and have lower reading anxiety (Zhao et al., 2013). However, having more opportunities to read English also means that they have to frequently deal with difficulties such as unfamiliar vocabulary and complicated sentence structures, which might increase anxiety. Therefore, international students face a greater challenge from demanding English reading in both study and life, which probably decreases their enjoyment and confidence and increases their anxiety when reading English.

In summary, similar extrinsic factors, long experience of English learning and similar postgraduate curriculums, mean that it is reasonable to suppose that the majority of advanced postgraduates would experience similar levels of FL reading anxiety, at a moderate level. However, the change of reading requirements and situations may lead to higher FL reading anxiety among Chinese postgraduates in the UK.

\subsection{The relationship between FL reading anxiety and reading performance}

By conducting the correlation analysis test, the present study found no statistically significant correlation between FLRAS scores and reading grades either in Group 1 or Group 2, or among participants across the groups, which indicates that FL reading anxiety had no influence on reading performance among the participants. In other words, their level of reading anxiety does not tend to influence their reading performance, which suggests that high anxiety students could possibly achieve high reading scores. The present study is not in line with the majority of research which has found a negative relationship between FL reading anxiety and reading performance (Mohammadpur et al., 2015; Saito et al., 1999; Sellers, 2000; Shi and Liu., 2006; Zin, 2010).

However, the results of the present study support the results of Brantmeier (2005) and Mills et al. (2006), and are partially consistent with the results of Joo et al. (2015) and Zhao et al. (2013). They found that no relationship existed between foreign language reading anxiety and reading performance. Possible factors leading to the lack of correlation could be interpreted in two ways: the difficulty of the reading tests and the unfamiliarity of the reading tests. Mills et al. (2006) suggested that the difficulty of the test may have been responsible for the insignificance of the result. It is possible that the CAE test has a higher difficulty level than other tests because it was designed for advanced English learners. Therefore, the difficulty for participants with advanced English or near advanced English level may be low. Thus, the difficulty of this reading test may not be the main factor for lack of correlation. In terms of the unfamiliarity of the reading test, a lack of previous practices with this test might also be an explanation. Regular and frequent practices, creating a familiarity with the reading task may reduce anxiety in reading (Brantmeier, 2005). If anxiety rose in the CAE reading, the reading processes might slow down due to the limited capacity of the working memory, which would further decrease reading efficiency and performance. Without regular, frequent and specific practice on the CAE reading test, low familiarity towards the unknown test format and new question types has a high probability of raising anxiety when reading this material. This may cause low reading grades and would account for the lack of correlation between reading anxiety and reading performance.

In the present study, the unfamiliarity of the reading test could be the major factor that reduced the correlation between FL reading anxiety and reading performance. Thus, the correlation between FL reading anxiety and reading anxiety still needs exploration in further research.

\subsection{Sources of reading anxiety}

The analysis of the interviews shows that reading anxiety sources differ, although international students and domestic students experienced similar levels of reading anxiety. Demanding text feature and different class settings induced anxiety in students in the UK, while students in China were negatively affected by lack of English exposure.

The change of the living and study environment may be an important reason causing reading anxiety for students in the UK. Specifically, they might lack enjoyment and have low confidence because of the high frequency of unknown words and having to analyse complicated sentences when reading. For example, words that are commonly used in the restaurant menus may not be familiar words to international students. In this way, it would be difficult to guess the meaning as there would be no prior knowledge so they would need to use dictionaries for extra help, impeding the word recognition process and the overall understanding of the context (Taguchi et al, 2004, p.88). The frequent interruption of word recognition process might induce anxiety when reading. Similarly, the sentences in the written materials are more likely to be complicated and elaborate, so international students may spend more time trying to understand English written materials (Gass, Behney, and Plonsky, 2013). Therefore, the unfamiliar language knowledge, countless time and effort spent reading in English could 
induce unpleasant feelings and anxiety among international students. This is in consistent with factors identified in the study by Saito et al. (1999), which indicated that unfamiliar scripts and writing systems might influence the normal procedures of reading processes and induce reading anxiety.

Apart from the potential anxiety caused by unfamiliar language knowledge, different learning styles and teaching approaches also induce anxiety in international students. This is somewhat in contrast to the factors identified by Shi and Liu. (2006), who pointed out that the teacher-centred approach in China increases students' dependence on teachers and diminishes their independent thinking and problem-solving abilities. In the UK, in contrast, the student-centred approach requires significant reading ability, understanding and critical thinking, attributes which are not actively developed in the teacher-centred approach in China. Interviewees in the UK referred to the fact that they were required to read many references and discussed them in class. However, due to their learning style, inculcated over many years, they may not have developed such strong understanding, interpreting and critical thinking ability as local students who were brought up in student-centred classrooms where active learning was the norm. Therefore, Chinese students in the UK might not enjoy the change of reading ability requirements embedded in the change in teaching approach and learning style.

However, demanding text feature and different class settings were not mentioned in the interviews of domestic students in China, where there are fewer opportunities to use English. A paucity of English learning opportunities, leading to the limited input of English and related knowledge could be the most influential factor causing reading anxiety (Larsen-Freeman and Long, 1991). The best way to learn a language is immersion in the target language, where comprehensible input is at the core of the language input (Krashen, 1989). Acquiring historical and cultural knowledge of the target language may help language learners understand reading materials (Huang, 2012, p.1521). Most participants in China (62.5\%) believed that English history and culture was useful when reading English, however, the participants, like most Chinese EFL learners have few opportunities to experience English and its related culture and history. Therefore, it is reasonable to suppose that they experience reading anxiety because of limited English exposure in China.

\section{Conclusion}

\subsection{Implications}

Based on the causes of reading anxiety in this study, recommendations are made for reducing the impact of factors leading to reading anxiety.

For international students, it is beneficial to develop appropriate attitudes towards significant change in their lifestyles and learning environment. Opportunities for using English increase because the living environment has changed from Chinese-dominated to English-dominated. In this way, students are required to be open-minded and make adjustments towards the target language and accept contradictions between their previous understanding and their newly developing knowledge of the target language. Second, by recognizing the purposes and requirements of the course in the UK in advance, students can practice and manage to change their ingrained reading style and learning habits in order to improve their independent-reading ability and critical thinking ability. In order to increase international students' understanding of local cultures and aid immersion into native living practices and studying environments, universities should hold regular specific cultural-experience activities and reading meetings. In this way, greater awareness of English culture and history could compensate for the deficiency caused by the bottom-up learning style. This greater awareness may help to reduce anxiety in foreign language reading.

As for home students, the lack of an immersive English learning environment is difficult to change in the short term. In light of this, postgraduates in China should be provided with more opportunities to take an active part in activities where English is used and required. For example, participating in English activities held by universities or social organizations, using applications to learn English online. While for English teachers in Chinese universities, more input and output opportunities should be created in the class by changing the traditional teacher-centred approach and helping students to play a more active role in class.

\subsection{Limitations}

First, the convenience sampling method, the relatively small sample size, the restricted subject of participants and a lack of gender balance may limit the generalizability of the study's findings, In other words, the results are not generalizable to the wider population of Chinese home students and international students. Thus, a larger sample size with gender balance and a variety of subjects is recommended for future research in the same area.

Second, the present study had only one reading test, which increased the potential influence of uncontrollable variables, such as physical features of participants, on the test results. Taking more tests would reduce uncertainty about reading 
grades and help to obtain a comprehensive account of students' reading performance, which would enhance the validity and reliability of the reading performance results.

Thirdly, the data of this study was collected over a short period of time. Combining with a longitudinal research project would be better to compensate the shortages of it, providing a more comprehensive investigation on different aspects of FL reading anxiety.

\section{Acknowledgments}

For the production and the preparation of this paper, I would like to express my sincere appreciation to my supervisor for her tremendous help. My supervisor consistently gave me valuable guidance, patiently answered my questions and led me into the correct direction when I needed it.

I would also like to acknowledge all the participants who were involved in the pilot study and data collection procedure. Without their enthusiastic participant, the survey could not have been successfully conducted.

I would also like to thank the proofreader of this paper. Thanks to his help, the paper was corrected and raised to a higher level of accuracy.

\section{References}

[1] Aida, Y. (1994). Examination of Horwitz, Horwitz, and Cope's construct of foreign language anxiety: The case of students of Japanese. The Modern Language Journal, 78(2), 155-168.

[2] Alderson, J. C., Haapakangas, E. L., Huhta, A., Nieminen, L., \& Ullakonoja, R. (2014). The diagnosis of reading in a second or foreign language. London: Routledge.

[3] Al-Shboul, M. M., Ahmad, I. S., Nordin, M. S., \& Rahman, Z. A. (2013). Foreign language reading anxiety in a Jordanian EFL context: A qualitative study. English Language Teaching, 6(6), 38-56.

[4] Biesta, G. (2017). Mixing methods in educational research. In R. Coe, M. Michael, L. V. Hedges, \& J. Arthur (Eds.), Research methods and methodologies in education (pp.159-165). London: SAGE Publications.

[5] Brantmeier, C. (2005). Anxiety about L2 reading or L2 reading tasks? A study with advanced language learners. The Reading Matrix, 5(2), 67-85.

[6] Breakwell, G. M., Smith, J. A. \& Wright, D. B. (2011). Research methods in psychology. London: Sage publications.

[7] Cambridge University Press. (2012). Cambriedge English: Advanced 5. Cambridge: Cambridge University Press.

[8] Cohen, L., Manion, L., \& Morrison, K. (2011). Research methods in education. London: Routledge.

[9] Coolican, H. (2013). Research methods and statistics in psychology. London: Hodder Education.

[10] Coward, H. (2007). Introducing short themed texts into the CAE reading paper. Cambridge ESOL: Research Notes. 30. 13-15.

[11] Eskey, D. E. (1998). Holding in the bottom: An interactive approach to the language problems of second language readers. In P. L. Carrell, J. Devine, \& D. E. Eskey (Eds.), Interactive approaches to second language reading (pp.93-100). Cambridge: Cambridge University Press.

[12] Gass, S. M., Behney, J., \& Plonsky, L. (2013). Second language acquisition: An introductory course. New York: Routledge.

[13] Grabe, W. (2009). Reading in a second language: Moving from theory to practice. Cambridge: Cambridge University Press.

[14] HESA. (2019). Higher education student statistics: UK, 2017/18 - Where students come from and go to study. Retrieved July 18, 2019 from The Higher Education Statistics Agency: https://www.hesa.ac.uk/news/17-01-2019/sb252-higher-education-student-statistics/location

[15] Horwitz, E. K., Horwitz, M. B., \& Cope, J. (1986). Foreign language classroom anxiety. The Modern Language Journal, 70(2), 125-132.

[16] Huang, Q. (2012). Study on correlation of foreign language anxiety and English reading anxiety. Theory and Practice in Language Studies, 2(7), 1520-1525.

[17] Isler, C., \& Yildirim, O. (2017). Sources of Turkish EFL learners' foreign language reading anxiety. JEELS: Journal of English Education and Linguistics Studies, 4(1), 1-18.

[18] Joo, K. Y., \& Damron, J. (2015). Foreign language reading anxiety: Korean as a foreign language in the United States. Journal of the National Council of Less Commonly Taught Languages, 17(1), 23-55.

[19] Khalifa, H., \& Schmitt, N. (2010). A mixed-method approach towards investigating lexical progression in main suite reading test papers. Cambridge ESOL: Research Notes. 41. 19-25.

[20] Krashen, S. D. (1989). Language acquisition and language education: Extensions and applications. New York: Prentice Hall International. 
[21] Larsen-Freeman, \& D., Long, M. H. (1991). An introduction to second language acquisition research. London: Longman.

[22] Larson-Hall, J. (2016). A guide to doing statistics in second language research using SPSS and R. London: Routledge.

[23] Lien, H. Y. (2016). Effects of EFL individual learner variables on foreign language reading anxiety and metacognitive reading strategy use. Psychological Reports, 119(1), 124-135.

[24] Lu, Z., \& Liu, M. (2015). An investigation of Chinese university EFL learner's foreign language reading anxiety, reading strategy use and reading comprehension performance. Studies in Second Language Learning and Teaching, 5(1), 65-85.

[25] MacIntyre, P. D. (1999). Language anxiety: A review of the research for language teachers. In Young, D. J. (Ed.) Affect in foreign language and second language learning: A practical guide to creating a low-anxiety classroom atmosphere, (pp. 24-41). London: McGraw Hill.

[26] Mills, N., Pajares, F., \& Herron, C. (2006). A reevaluation of the role of anxiety: Self - efficacy, anxiety, and their relation to reading and listening proficiency. Foreign language annals, 39(2), 276-295.

[27] Ministry of Education of The People's Republic of China. (2001). MOE guidelines for actively promote the revolution on the assessment system in elementary and secondary school. Retrieved July 26, 2019 from http://www.moe.gov.cn/ srcsite/A26/s7054/200101/t20010120_166075.html

[28] Mohammadpur, B., \& Ghafournia, N. (2015). An Elaboration on the Effect of Reading Anxiety on Reading Achievement. English Language Teaching, 8(7), 206-215.

[29] Nuttall, C. (2005). Teaching reading skills in a foreign language. Oxford: Macmillan Education.

[30] Perfetti, C., \& Harris, L. (2013). Universal reading processes are modulated by language and writing system. Language Learning and Development, 9(4), 296-316.

[31] Robson, C., \& McCartan, K. (2011). Real world research. Chichester: Wiley.

[32] Saito, Y., Garza, T. J., \& Horwitz, E. K. (1999). Foreign language reading anxiety. The Modern Language Journal, 83(2), 202-218.

[33] Samuels, S. J., \& Kamil, M. L. (1998). Models of the reading process. In P. L. Carrell, J. Devine, \& D. E. Eskey (Eds.), Interactive approaches to second language reading (pp.22-36). Cambridge: Cambridge University Press.

[34] Sasaki, M. (2005). The effect of L1 reading processes on L2: A crosslinguistic comparison of Italian and Japanese users of English. In V. Cook, \& B. Bassetti (Eds.), Second language writing systems (pp.289-308). Clevedon: Multilingual Matters.

[35] Sellers, V. D. (2000). Anxiety and reading comprehension in Spanish as a foreign language. Foreign Language Annals, 33(5), 512-520.

[36] Shi, Y. Z., \& Liu, Z. Q. (2006). Foreign Language Reading Anxiety and its relationship to English achievement and gender. Journal of PLA University of Foreign Languages, 29(2), 59-64.

[37] Taguchi, E., Takayasu-Maass, M., \& Gorsuch, G. J. (2004). Developing reading fluency in EFL: How assisted repeated reading and extensive reading affect fluency development. Reading in a Foreign Language, 16(2), 70-96.

[38] Zhao, A., Guo, Y., \& Dynia, J. (2013). Foreign language reading anxiety: Chinese as a foreign language in the United States. The Modern Language Journal, 97(3), 764-778.

[39] Zhou, J. (2017). Foreign language reading anxiety in a Chinese as a foreign language context. Reading in a Foreign Language, 29(1), 155-173.

[40] Zin, M. (2010). Anxiety and Academic Reading Performance among Malay ESL Learners. Journal of Pan-Pacific Association of Applied Linguistics, 14(2), 41-58. 